\title{
Preconditioning of Low-Frequency Repetitive Transcranial Magnetic Stimulation with Transcranial Direct Current Stimulation: Evidence for Homeostatic Plasticity in the Human Motor Cortex
}

\author{
Hartwig R. Siebner, ${ }^{1,2}$ Nicolas Lang, ${ }^{1,3}$ Vincenzo Rizzo,,${ }^{1,4}$ Michael A. Nitsche, ${ }^{3}$ Walter Paulus, ${ }^{3}$ Roger N. Lemon, ${ }^{1}$ and \\ John C. Rothwell ${ }^{1}$ \\ ${ }^{1}$ Sobell Department of Motor Neuroscience and Movement Disorders, Institute of Neurology, University College of London, London WC1N 3BG, United \\ Kingdom, ${ }^{2}$ Department of Neurology, Christian-Albrechts University, 24105 Kiel, Germany, ${ }^{3}$ Department of Clinical Neurophysiology, Georg-August \\ University, 37075 Göttingen, Germany, and ${ }^{4}$ Department of Neurosciences and Psychiatric and Anaesthesiological Sciences, University of Messina, 98125 \\ Messina, Italy
}

Recent experimental work in animals has emphasized the importance of homeostatic plasticity as a means of stabilizing the properties of neuronal circuits. Here, we report a phenomenon that indicates a homeostatic pattern of cortical plasticity in healthy human subjects. The experiments combined two techniques that can produce long-term effects on the excitability of corticospinal output neurons: transcranial direct current stimulation (TDCS) and repetitive transcranial magnetic stimulation (rTMS) of the left primary motor cortex. "Facilitatory preconditioning" with anodal TDCS caused a subsequent period of $1 \mathrm{~Hz}$ rTMS to reduce corticospinal excitability to below baseline levels for $>20 \mathrm{~min}$. Conversely, "inhibitory preconditioning" with cathodal TDCS resulted in $1 \mathrm{~Hz}$ rTMS increasing corticospinal excitability for at least $20 \mathrm{~min}$. No changes in excitability occurred when $1 \mathrm{~Hz}$ rTMS was preceded by sham TDCS. Thus, changing the initial state of the motor cortex by a period of DC polarization reversed the conditioning effects of $1 \mathrm{~Hz}$ rTMS. These preconditioning effects of TDCS suggest the existence of a homeostatic mechanism in the human motor cortex that stabilizes corticospinal excitability within a physiologically useful range.

Key words: corticospinal excitability; homeostatic plasticity; human motor cortex; transcranial magnetic stimulation; transcranial direct current stimulation; metaplasticity

\section{Introduction}

Over the last 25 years, bidirectional changes in synaptic strength driven by neuronal activity have emerged as a powerful mechanism for tuning the response properties of cortical neurons in the mammalian brain (Bear and Malenka, 1994). Activity-dependent synaptic plasticity, such as long-term potentiation (LTP) and long-term depression (LTD), is also expressed in the motor cortex (Iriki et al., 1989; Hess and Donoghue, 1996) and is thought to play an important role in motor learning (Asanuma and Keller, 1991; Rioult-Pedotti et al., 2000).

Because of its positive-feedback nature, activity-driven synaptic plasticity carries the risk of destabilizing the properties of neuronal networks (Abbott and Nelson, 2000; Turrigiano and Nelson, 2004). Therefore, it has long been postulated that regulatory mechanisms must exist to stabilize neuronal activity within a

Received Nov. 13, 2003; revised Feb. 23, 2004; accepted Feb. 23, 2004

H.R.S. and N.L. were supported by the Deutsche Forschungsgemeinschaft. H.R.S. is currently supported by a Bundesministerium für Bildung und Forschung grant (GF G0 1337400).

Correspondence should be addressed to Dr. Hartwig Roman Siebner, Department of Neurology, ChristianAlbrechts University Kiel, Niemannsweg 147, D-24105 Kiel, Germany. E-mail: h.siebner@neurologie.uni-kiel.de. D0I:10.1523/JNEUROSC1.5316-03.2004

Copyright $\odot 2004$ Society for Neuroscience $\quad 0270-6474 / 04 / 243379-07 \$ 15.00 / 0$ useful dynamic range (Sejnowski, 1977). A theoretical solution to this stability problem was provided by the Bienenstock-CooperMunro (BCM) rule of synaptic modification, which was proposed to explain the development of stimulus selectivity in the visual cortex (Bienenstock et al., 1982). The BCM rule says that stabilization of neuronal activity is ensured by a dynamic adaptation of the "modification threshold" (the level of postsynaptic response below, which gives $\mathrm{LTD}$, and above, which gives LTP) to the time-averaged value of the postsynaptic activity. For example, a prolonged reduction in postsynaptic activity would reduce the modification threshold, favoring the induction of LTP. Conversely, a prolonged increase in postsynaptic activity would raise the modification threshold, favoring the induction of LTD.

Confirming the BCM rule, recent animal research has disclosed several homeostatic mechanisms in the hippocampus and neocortex that regulate synaptic plasticity according to the previous history of neural activity (Huang et al., 1992; Kirkwood et al., 1996; Wang and Wagner, 1999): high levels of activity favor synaptic depression, whereas low levels of activity favor facilitation. This "homeostatic plasticity" enables selective modification of synaptic strength while maintaining total synaptic strength within a physiological range (Abraham and Tate, 1997; Abbott 
and Nelson, 2000; Turrigiano and Nelson, 2004). Given these studies in animals, it seems reasonable to assume that homeostatic plasticity will play an important regulatory role in the human cortex as well.

In the present experiments, we interleaved repetitive transcranial magnetic stimulation (rTMS) with transcranial direct current stimulation (TDCS) to disclose homeostatic plasticity in the human motor cortex. Both stimulation techniques can produce bidirectional aftereffects on the excitability of corticospinal output neurons depending on the frequency (rTMS) or polarity (TDCS) of stimulation (Pascual-Leone et al., 1994; Chen et al., 1997; Maeda et al., 2000a; Nitsche and Paulus, 2001; Touge et al., 2001; Nitsche et al., 2003). Although the exact mechanisms mediating these aftereffects remain to be clarified, there is some evidence that changes in synaptic efficacy play a part (Liebetanz et al., 2002; Iyer et al., 2003; Siebner and Rothwell, 2003). According to the BCM rule, we hypothesized that "facilitatory" preconditioning with anodal TDCS will favor a subsequent period of $1 \mathrm{~Hz}$ rTMS to reduce corticospinal excitability. Conversely, we expected that "inhibitory" preconditioning with cathodal TDCS will increase the ability of $1 \mathrm{~Hz}$ rTMS to enhance corticospinal excitability.

\section{Materials and Methods}

Subjects. Eight healthy male volunteers (mean age, 35 years; age range, 27-47 years) gave their written informed consent to participate in the study. Seven subjects were consistent right-handers, and one subject was a consistent left-hander (Oldfield, 1971). Experimental procedures were approved by the Joint Ethics Committee of the National Hospital for Neurology and Neurosurgery and the Institute of Neurology and performed according to the ethical standards laid down in the Declaration of Helsinki. Subjects were seated in a comfortable reclining chair with a mounted headrest during experiments. None of the subjects reported adverse effects during the course of the study.

Experimental approach. In the main experiment, we explored the effect of TDCS priming on the conditioning effect of $1 \mathrm{~Hz}$ rTMS on motor cortex excitability (Fig. 1). It has been shown that both techniques are capable of increasing or decreasing the excitability of corticospinal output neurons depending on the polarity (TDCS) or frequency (rTMS) of stimulation (Pascual-Leone et al., 1994; Chen et al., 1997; Nitsche and Paulus, 2001; Nitsche et al., 2003). All participants received 15 min of subthreshold $1 \mathrm{~Hz}$ rTMS to the left primary motor cortex (M1), which may sometimes produce a weak suppression of corticospinal excitability (Maeda et al., 2000b; Fitzgerald et al., 2002). This was preceded either by a 10 min period of effective TDCS to the left M1 using anodal (excitatory) or cathodal (inhibitory) polarity or by sham TDCS. Corticospinal excitability, short-latency intracortical inhibition, and short-latency intracortical facilitation were measured with TMS over the left M1 before TDCS (referred to as baseline), after TDCS, and twice after rTMS (Kujirai et al., 1993). The excitability of the left M1 was assessed in separate blocks that lasted $\sim 10$ min (Fig. 1)

Five of the eight subjects participated in a control experiment, which was designed to assess changes in excitability when TDCS to the left M1 was administered alone (Fig. 1). The same subjects took part in a second control experiment, in which TDCS was administered to the left posterior parietal cortex. We delivered TDCS to a parietal site posterior to M1 to test the topographic specificity of the preconditioning effects of TDCS.

rTMS. During $1 \mathrm{~Hz}$ rTMS, each magnetic stimulus generates a pulsed magnetic field to induce an electrical current in the underlying brain (Rothwell, 1997; Hallett, 2000). We used a rapid stimulator (Magstim, Whitland, Wales, UK) and a standard figure-of-eight coil (Magstim) for $1 \mathrm{~Hz}$ rTMS. The settings for rTMS were identical for each experiment. The coil was placed tangentially to the scalp, with the handle pointing posterolaterally at a $45^{\circ}$ angle from the midline. The site where biphasic stimuli of slightly suprathreshold intensity consistently produced the largest motor-evoked potentials (MEPs) with the steepest negative slope in the right first dorsal interosseus (FDI) muscle (referred to as the "mo-
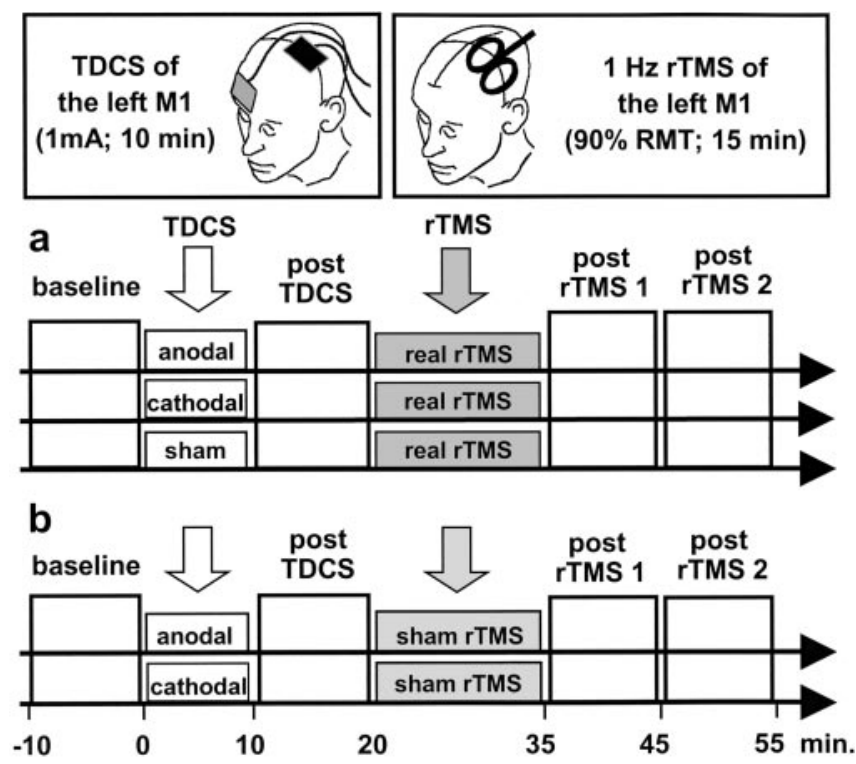

Figure 1. Experimental design. $a$, In the main experiment, a 10 min session of anodal, cathodal, or sham TDCS was given to the left primary motor hand area (M1) on separate days. For anodal TDCS, the anode was placed over the left M1, and the cathode was placed over the right eyebrow. Polarity was reversed for cathodal TDCS. At $10 \mathrm{~min}$ after the end of the TDCS session, 900 biphasic pulses of $1 \mathrm{~Hz}$ rTMS were given to the left M1 at $85 \%$ of resting motor threshold. Corticospinal excitability was probed with single-pulse and paired-pulse TMS over the left M1 before TDCS, after TDCS, and twice after rTMS. $b$, In the control experiment, the conditioning effects of anodal and cathodal TDCS alone were explored in five subjects (10 min, $1 \mathrm{~mA}$ ). To control for nonspecific effects of rTMS, real rTMS was replaced by sham rTMS using a specifically designed placebo coil.

tor hot spot") was marked on the scalp. Resting motor threshold (RMT) was assessed as the lowest intensity that was able to evoke an MEP of $>50$ $\mu \mathrm{V}$ in at least 5 of 10 consecutive trials in the right FDI muscle.

A number of studies have shown that $1 \mathrm{~Hz}$ rTMS to the M1 can induce a lasting decrease in corticospinal excitability. However, the decrease in excitability is seen more prominently and for a longer time if the intensity of the rTMS is above RMT (Chen et al., 1997; Muellbacher et al., 2000) rather than at or below RMT, when the effects are much less consistent (Siebner et al., 1999b; Maeda et al., 2000a; Touge et al., 2001; Fitzgerald et al., 2002; Romero et al., 2002; Sommer et al., 2002). In the present study, we used an intensity of $85 \%$ of RMT for two reasons: First, the intensity is likely to activate corticospinal neurons trans-synaptically without causing a direct activation of the corticospinal neurons (Di Lazzaro et al., 1999). Second, we reasoned that it would produce a relatively "weak" aftereffect that would be more susceptible to the effects of pre-conditioning.

The mean intensity of rTMS (given as a percentage of maximum stimulator output) was matched among sessions $(50 \pm 3 \%$ after anodal TDCS, $49 \pm 3 \%$ after cathodal TDCS, and $48 \pm 3 \%$ after sham TDCS). In the control experiment, sham rTMS was given through a specially designed sham coil that induced no magnetic field but evoked a comparable acoustic artifact (Magstim).

TDCS. TDCS involves continuous administration of weak currents of $\sim 1 \mathrm{~mA}$ through a pair of surface electrodes attached to the scalp (Nitsche and Paulus, 2000). In contrast to phasic suprathreshold stimulation of cortical neurons evoked by rTMS, TDCS causes a tonic subthreshold facilitation or inhibition of cortical neurons depending on the polarity of the current. On the basis of animal studies published in the 1960s (Bindman et al., 1962, 1964; Gartside, 1968), it has been proposed that the primary mechanism of action of TDCS is a polarity-specific shift of resting membrane potentials in cortical neurons resulting in secondary alterations of spontaneous discharge rates.

Continuous TDCS of the M1 used a battery-driven DC stimulator (Schneider Electronic, Gleichen, Germany). For effective TDCS of the $\mathrm{M} 1$, a constant current flow of $1 \mathrm{~mA}$ was applied through wet sponge 
electrodes $(7 \times 5 \mathrm{~cm}$ in size $)$ placed over the left $\mathrm{M} 1$ and the right frontal pole (Fig. 1). The M1 electrode was placed at the optimal site for cortical magnetic stimulation. The frontopolar electrode was always placed over the right eyebrow. TDCS polarity refers to the electrode placed over the left M1. For anodal TDCS, the anode was placed over the M1, whereas the cathode was over the M1 during cathodal TDCS.

In the first control experiment, we gave sham TDCS to the left M1. For sham TDCS, the DC stimulator was only switched on for $5 \mathrm{sec}$ at the beginning of the sham session and then turned off. In the second control experiment, the left posterior parietal cortex was preconditioned with TDCS. For parietal TDCS, the electrodes were placed $5 \mathrm{~cm}$ posterior to the M1 (parietal electrode) and over the right eyebrow (frontopolar electrode). Otherwise, procedures were identical to effective TDCS of the left M1.

Recording technique. Measurements of corticospinal excitability were made in separate blocks before TDCS, after TDCS, as well as two times after $1 \mathrm{~Hz}$ rTMS (Fig. 1). In all participants, MEPs were recorded through a pair of $\mathrm{Ag}-\mathrm{AgCl}$ surface electrodes placed over the right FDI muscle, using a belly-tendon montage. Raw signals were amplified and bandpass filtered ( $3 \mathrm{~Hz}$ to $1 \mathrm{kHz}$ ). Signals were digitized using a CED 1401 laboratory interface (Cambridge Electronic Design, Cambridge, UK) and stored at a sample rate of $5 \mathrm{kHz}$. Auditory (speakers) and visual (oscilloscope) feedback of EMG activity were given to the subjects to ensure complete relaxation.

The paired-pulse technique described by Kujirai et al. (1993) was used to probe changes in both corticospinal excitability and intracortical excitability of the left M1. Paired magnetic pulses were generated by two high-powered Magstim 200 stimulators connected by a Bistim module and delivered through a standard figure-of-eight coil with a $9 \mathrm{~cm}$ outer diameter of each wing placed over the motor hot spot of the left M1 (Magstim). The magnetic stimulus had a nearly monophasic pulse configuration with a rise time of $\sim 100 \mu \mathrm{sec}$. The coil current during the rising phase of the magnetic field flowed toward the handle. The coil was placed tangentially to the scalp, with the handle pointing backward and laterally at a $45^{\circ}$ angle to the sagittal plane, inducing a posteroanterior current in the brain.

The intensity of the conditioning stimulus was set to $80 \%$ of active motor threshold (AMT). AMT was defined as the stimulator intensity sufficient to elicit a reliable MEP of at least $200 \mu \mathrm{V}$ in amplitude in the tonically contracting contralateral FDI muscle in at least 5 of 10 consecutive stimuli. The test stimulus was set at an intensity that, when given alone, would evoke an EMG response of 0.7-1 mV peak-to-peak amplitude in the relaxed contralateral FDI muscle. In addition to the unconditioned test stimulus, paired pulses were given at four conditioning-test intervals. Interstimulus intervals (ISIs) of 2 and 4 msec probed the magnitude of short-latency intracortical inhibition, whereas ISIs of 9 and 12 msec were used to assess the strength of short-latency intracortical facilitation. In each block, we recorded 15 trials for each paired-pulse condition and 30 trials for the test stimulus alone in a pseudorandom order. The intertrial interval was set at $5 \mathrm{sec}$.

Data analysis. For each block of measurements, the peak-to-peak amplitudes of each MEP (in millivolts) were measured off-line, and the mean MEP amplitudes were calculated for each stimulation condition. MEP amplitudes were normalized to preintervention values and entered in a three-way repeated-measures ANOVA with time of measurement (i.e., block of trials), type of intervention (anodal TDCS, cathodal TDCS, and sham TDCS), and measure of cortical excitability (single-pulse TMS, short-latency intracortical inhibition, and short-latency intracortical facilitation) as within-subject factors. The Greenhouse-Geisser method was used when necessary to correct for nonsphericity. A $p$ value of $<0.05$ was considered significant. Data are given as means \pm SEM.

\section{Results}

TDCS conditioning caused a polarity-specific change in corticospinal excitability. Ten minutes of anodal TDCS induced an increase in mean MEP amplitude, whereas cathodal TDCS produced a decrease in mean MEP amplitude. However, the magnitude of change in corticospinal excitability was moderate and variable among participants, and neither anodal nor cathodal TDCS induced a change in MEP amplitude that was significantly different from baseline (paired Student's $t$ test; anodal TDCS, $p=0.08$; cathodal TDCS, $p=0.37$ ). Nevertheless, the preconditioning TDCS had a profound impact on the conditioning effects induced by subsequent administration of $1 \mathrm{~Hz}$ rTMS (Fig. 2a). When the excitability of the corticospinal projection had been raised by 10 min of anodal TDCS, a subsequent period of $1 \mathrm{~Hz}$ rTMS reduced corticospinal excitability for at least 20 min after the end of rTMS. Conversely, when cathodal TDCS had been used to reduce corticospinal excitability, the same $1 \mathrm{~Hz}$ rTMS increased corticospinal excitability. These effects led to a significant interaction between the factors time and intervention $\left(F_{(2.7,18.9)}=6.3 ; p=0.005\right)$ in the ANOVA.

Analysis of the data from individual subjects showed that there was a significant inverse correlation between the magnitude of effects immediately after TDCS and the subsequent aftereffect of rTMS. Subjects who had the largest increase in excitability after anodal TDCS showed the greatest depression after rTMS (Pearson's correlation; $r=-0.86 ; p=0.006$ ). Similarly, subjects with the largest reduction in excitability after cathodal TDCS had the largest increase after rTMS (Pearson's correlation; $r=-0.75$; $p=0.03$ ).

The effects of rTMS on corticospinal excitability outweighed the preconditioning effects of TDCS, resulting in a relative change in excitability compared with baseline levels. Indeed, an ANOVA limited to the comparison of baseline and the post$\mathrm{rTMS}_{2}$ measurements revealed an interaction between time and intervention $\left(F_{(1.1,8.0)}=7.2 ; p=0.026\right)$ attributable to the opposite sign of rTMS-induced excitability changes after anodal and cathodal pre-conditioning.

We also evaluated the amount of short-latency intracortical inhibition and short-latency intracortical facilitation at the same time points. Anodal or cathodal TDCS did not change the relative magnitude of short-latency intracortical inhibition or shortlatency intracortical facilitation, and neither did subsequent administration of $1 \mathrm{~Hz}$ rTMS (Fig. 3). Hence, the sign and strength of stimulation-induced changes were comparable for each measure of cortical excitability.

In a first control experiment on five subjects, we explored changes in excitability when TDCS was given alone (Fig. 2b). In accordance with previous reports (Nitsche and Paulus, 2001; Nitsche et al., 2003), anodal TDCS induced a long-lasting increase in corticospinal excitability, whereas cathodal TDCS resulted in a sustained decrease in corticospinal excitability. Polarity-specific aftereffects on corticospinal excitability account for a significant time by intervention interaction $\left(F_{(1.6,6.5)}=11.5\right.$; $p=0.009)$ in the ANOVA. The conditioning effects induced by TDCS alone were stable for at least $45 \mathrm{~min}$ (Fig. $2 b$ ).

A second control experiment revealed that the preconditioning effects of TDCS were specific to TDCS over the left M1. Preconditioning over the left posterior parietal cortex failed to induce a bidirectional modulation in corticospinal excitability (Fig. $2 c$ ) or to produce a preconditioning effect on subsequent rTMS conditioning of the left M1 (Fig. 2c). This was confirmed by the ANOVA, which showed neither a main effect for time or intervention nor a time by intervention interaction.

\section{Discussion}

The present results show that preconditioning corticospinal excitability with TDCS over the M1 can modulate the direction of plasticity induced by subsequent administration of $1 \mathrm{~Hz}$ rTMS. These pre-conditioning effects were regionally specific to TDCS over the left M1, because TDCS administered to the left posterior 


\section{a Main experiment $(\mathrm{n}=8)$}

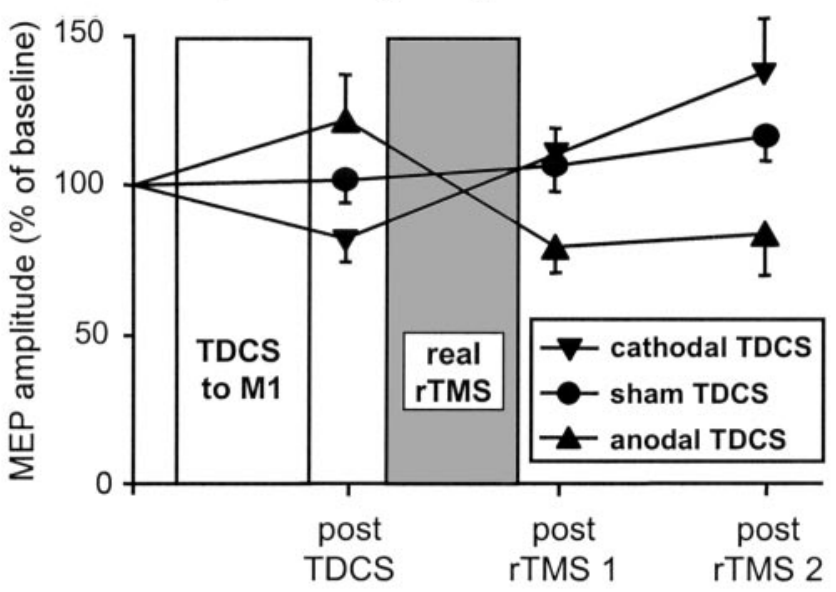

\section{b First control experiment $(n=5)$}

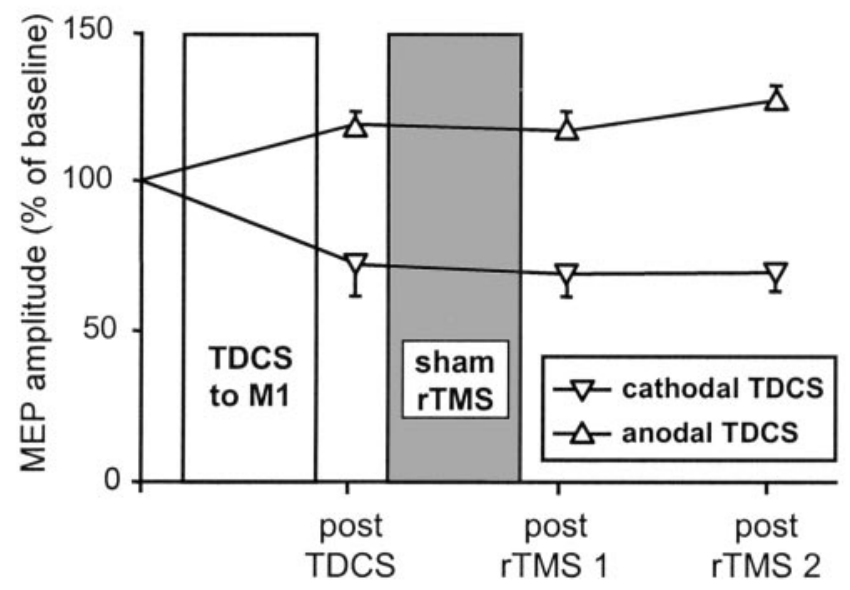

\section{Second control experiment $(n=5)$}

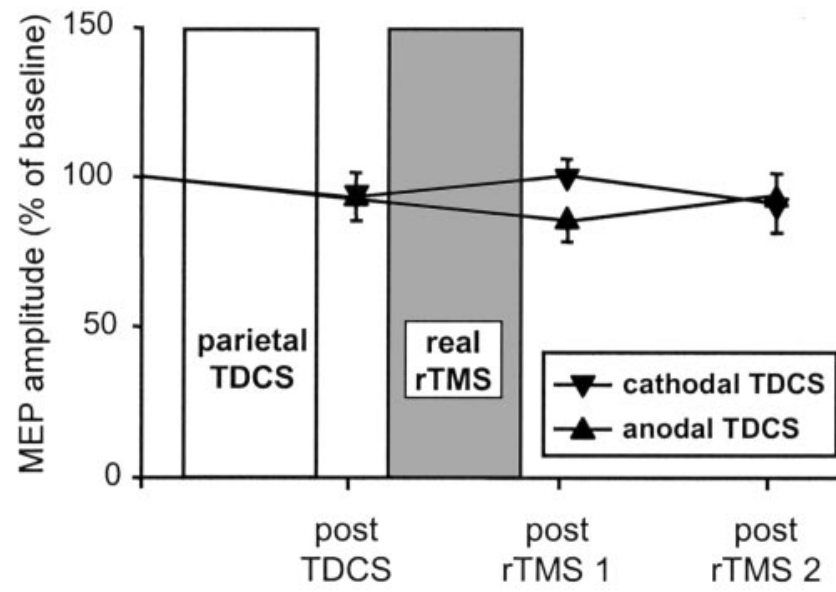

Figure2. Stimulation-induced changes in motor cortical output to the right first dorsal interosseus muscle. $a$, In the main experiment, the figure plots the amplitude of a standard test MEP evoked by a single TMS probe stimulus at different times before and after the two types of conditioning. The aftereffects of $1 \mathrm{~Hz}$ rTMS were critically dependent on the preconditioning by TDCS over the left M1. A total of $1 \mathrm{~Hz}$ rTMS reversed polarity-specific effects induced by anodal or cathodal TDCS. Sham TDCS followed by rTMS had no impact on corticospinal excitability. $b$, In the first control experiment, TDCS alone induced a polarity-specific shift in the level of corticospinal excitability. Anodal TDCS caused a sustained increase in excitability, whereas cathodal TDCS resulted in a lasting reduction of corticospinal excitability. c, In the second control experiment, when TDCS was administered to the left posterior parietal cortex, TDCS produced no polarity-specific shift in ipsilateral corticospinal excitability and had parietal cortex failed to produce any consistent preconditioning effect on subsequent administration of $1 \mathrm{~Hz}$ rTMS to the M1. When the excitability level of the corticospinal projection had been raised by $10 \mathrm{~min}$ of anodal TDCS to the left M1, a subsequent period of $1 \mathrm{~Hz}$ rTMS led to a lasting reduction in corticospinal excitability. Conversely, when cathodal TDCS was used to reduce corticospinal excitability, the same $1 \mathrm{~Hz}$ rTMS caused a sustained increase in corticospinal excitability. Those subjects who had the largest aftereffects from TDCS also had the largest changes in the response to rTMS. This pattern is exactly what is expected from a homeostatic mechanism that controls excitability in the human corticospinal system and acts to stabilize excitability levels within a physiologically useful modification range.

Here we used, relatively speaking, a fairly short train and a weak stimulus that produced no consistent effect on corticospinal excitability. We think that giving more pulses or higher intensity would have produced a clearer effect on corticospinal excitability (Chen et al., 1997; Muellbacher et al., 2000), but in the present experiment, the lack of a significant effect made the interpretation of the data much simpler. A recent study demonstrated that a priming session of high-frequency rTMS at $6 \mathrm{~Hz}$ can be used to enhance the effect of subsequent administration of 1 $\mathrm{Hz}$ rTMS on corticospinal excitability, producing a stronger suppression of corticospinal excitability (Iyer et al., 2003). In the present study, a similar effect was observed when anodal TDCS preceded $1 \mathrm{~Hz}$ rTMS. However, the critical new finding is that preconditioning with cathodal TDCS reversed the direction of rTMS-induced plasticity, causing a lasting increase in corticospinal excitability.

The homeostatic priming effects of TDCS on motor cortex plasticity are analogous to homeostatic plasticity as described in animal preparations, in which the magnitude and direction of synaptic plasticity are adjusted according to the recent history of postsynaptic activity (Huang et al., 1992; Kirkwood et al., 1996; Wang and Wagner, 1999). Homeostatic plasticity has been demonstrated in slice preparations of the hippocampus (Huang et al., 1992; Bear, 1996; Wang and Wagner, 1999) or visual cortex (Kirkwood et al., 1996) of the rat and the amygdala of the guinea pig (Royer and Pare, 2003). For example, in hippocampal slices, high-frequency bursts of a priming stimulus that has no longterm effects on synaptic efficacy itself can reverse the effect of a 10 $\mathrm{Hz}$ conditioning protocol from excitatory to inhibitory (Wang and Wagner, 1999). The effect is present $45 \mathrm{~min}$ after the priming stimulus and affects both homosynaptic input (i.e., the priming and conditioning stimuli activate the same synaptic inputs) and heterosynaptic input (Wang and Wagner, 1999). The results can be interpreted in terms of the BCM model of activity-dependent shifts in synaptic plasticity. Thus, the high-frequency priming stimulus gives the neurons a history of high excitation, and this leads to a shift in the frequency response of LTP and LTD conditioning protocols to favor LTD.

The first control experiment revealed that TDCS alone induced a bidirectional shift in corticospinal excitability depending on the polarity of TDCS. The implication is that homeostatic plasticity did not prevent the induction of persistent changes in

$\leftarrow$

no preconditioning effect on subsequent administration of $1 \mathrm{~Hz}$ rTMS to the left M1. In each panel, the symbols represent the mean MEP amplitude (normalized to the mean MEP amplitude at baseline) after TDCS (first column) and after rTMS (second and third columns). Error bars represent SEM. Both control experiments were conducted in five of the eight subjects who had participated in the main experiment. 


\section{a Anodal TDCS followed by rTMS}

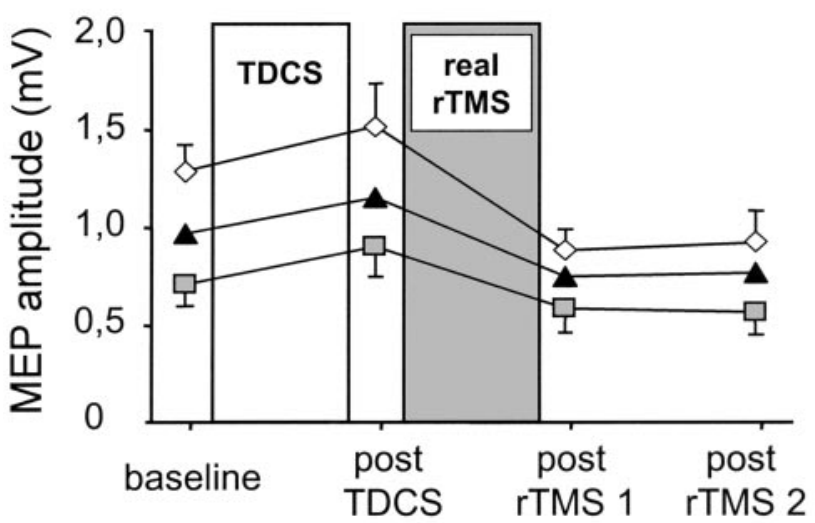

\section{b Cathodal TDCS followed by rTMS}

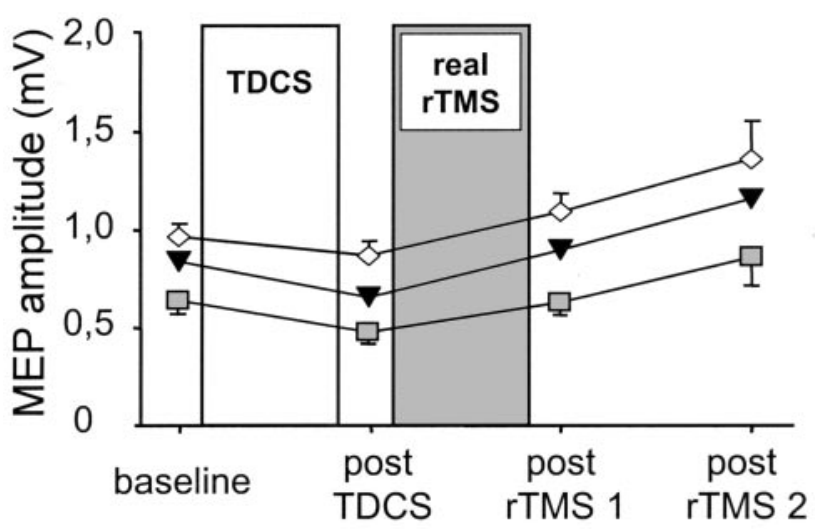

$$
\begin{aligned}
& \multimap \text { Paired TMS at ISIs of } 9 \text { and } 12 \text { ms (SICF) } \\
& \leftarrow \text { Single-pulse TMS (Corticospinal excitability) } \\
& \neg-\text { Paired TMS at ISIs of } 2 \text { and } 4 \text { ms (SICI) }
\end{aligned}
$$

Figure 3. Stimulation-induced effects on various measures of motor cortical excitability. Motor cortical excitability was assessed with single-pulse TMS and paired-pulse TMS at different ISIs using a conditioning-test paradigm (Kujirai et al., 1993). This allowed for assessment of changes in corticospinal excitability (single magnetic pulses) and intracortical excitability of the left M1 (pairs of magnetic pulses). ISIs of 2 and 4 msec probed the magnitude of short-latency intracortical inhibition (SICI), whereas ISIs of 9 and 12 msec were used to assess the strength of short-latency intracortical facilitation (SICF). Changes in paired-pulse excitability paralleled changes in single-pulse excitability. $a$ illustrates the effects of anodal TDCS followed by $1 \mathrm{~Hz}$ rTMS. $b$ presents the effects of cathodal TDCS followed by $1 \mathrm{~Hz}$ rTMS. Symbols and error bars represent the mean MEP amplitude and SEM, respectively.

excitability at the time of preconditioning (i.e., TDCS session) but only counteracted an additional shift in excitability during subsequent conditioning (i.e., rTMS session). We suppose that at the time of preconditioning, homeostatic mechanisms were "idling," because the motor cortex was more or less close to the center of its modification range. Therefore, TDCS was capable of inducing some plastic change in excitability, which then called homeostatic plasticity into action.

In recent years, several studies on cortical slices in rats have shown that homeostatic plasticity plays a critical role in the control of synaptic plasticity in vivo. In these studies, plasticity induced by motor learning or sensory deprivation changed the synaptic modification threshold in the motor (Rioult-Pedotti et al.,
2000), visual (Desai et al., 2002), and somatosensory (Allen et al., 2003) cortex according to the BCM rule. For instance, motor skill training resulted in a marked reduction of LTP and an enhancement of LTD in the trained M1 compared with the untrained M1 (Rioult-Pedotti et al., 2000). Our results are similar to these animal data; indeed, we found that two different priming protocols (anodal or cathodal TDCS) produced opposite effects on the subsequent conditioning rTMS trains in the human corticospinal motor system.

Without additional invasive experiments on the primate brain, we can only speculate on the precise neuronal mechanisms that may underlie the effect we observed. When given for a period longer than $\sim 5 \mathrm{~min}$, DCs applied directly to the surface of the rat cortex cause both immediate and long-lasting changes in the level of activity of pyramidal neurons: discharge is increased by anodal polarization or decreased by cathodal polarization (Bindman et al., 1962, 1964; Purpura and McMurry, 1965; Gartside, 1968). Our model is as follows: in the human motor cortex, a TDCSinduced change in postsynaptic activity of cortical neurons causes an activity-dependent adjustment of the long-term modification threshold for (synaptic) plasticity induced by rTMS. The increased postsynaptic activity produced by anodal TDCS would favor depressive effects of rTMS, whereas the decrease in activity produced by cathodal TDCS would favor facilitatory effects.

Direct recordings of the corticospinal volley evoked by TMS from the cervical epidural space suggest that with the coil and intensity we used, single and paired pulses induced mainly indirect (trans-synaptic) stimulation of corticospinal neurons with very little direct activation (Di Lazzaro et al., 1999). Although TMS can directly activate corticospinal neurons at higher stimulus intensities, the latencies of directly evoked corticospinal volleys indicate activation at some distance from the cell body down the axon, where the evoked descending volley in the corticospinal pyramidal tract is not influenced by the level of cortical excitability (Di Lazzaro et al., 1999). Therefore, we favor the hypothesis that the homeostatic mechanism involved an altered integration of synaptic inputs in dendrites, or at the cell body of corticospinal neurons, or a shift in membrane excitability.

The adjustment of modification threshold might occur in corticospinal output neurons themselves, be mediated by intracortical neurons that project onto the corticospinal neurons, or both. We used a conditioning-test paradigm (Kujirai et al., 1993) to study the effects of TDCS preconditioning on the balance between intracortical facilitatory and inhibitory circuits. Because we found no differential effect on the magnitude of short-latency intracortical inhibition and facilitation, our results favor a direct homeostatic effect in the corticospinal output neurons rather than a homeostatic mechanism within intracortical interneuronal circuits. Using rTMS protocols that selectively change the excitability of distinct intracortical circuits in M1 (Di Lazzaro et al., 2002; Munchau et al., 2002) might help to clarify the underlying mechanisms. Additionally, pharmacological modulation of the preconditioning effects might also help to shed some light on the mechanisms involved in this form of homeostatic plasticity. However, these drugs need to be short acting to selectively interact with preconditioning (TDCS) but not with conditioning (rTMS).

Whatever the neuronal mechanism of the priming effect of TDCS, to our knowledge, this is the first study to provide experimental evidence for the existence of a BCM-like mechanism of homeostatic plasticity that can acutely regulate excitability in the human corticospinal motor system. In a rat model of parkinsonism, L-3,4-dihydroxyphenylalanine-induced dyskinesia is associ- 
ated with abnormal homeostatic regulation of LTP in the striatum (Picconi et al., 2003). This suggests that an impairment of homeostatic plasticity may be of relevance in the pathophysiology of brain diseases. Our experimental approach provides a paradigm to probe in the intact human brain whether and how patients with neuropsychiatric diseases express aberrant forms of homeoplastic plasticity in the corticospinal motor system. Because measurements can be repeated over time, it is also possible to explore how therapeutic interventions shape homeostatic plasticity and how this is related to therapeutic efficacy.

The suppressive effect of $1 \mathrm{~Hz}$ rTMS on cortical excitability has been used to induce a temporary cortical dysfunction in healthy subjects (Kosslyn et al., 1999; Hilgetag et al., 2001; Muellbacher et al., 2002; Knecht et al., 2003) or, presumably by normalizing increased levels of excitability, to induce beneficial effects in patients with neuropsychiatric disorders (Klein et al., 1999; Siebner et al., 1999b; Tergau et al., 1999; Brighina et al., 2003; Hoffman et al., 2003). Here, we found that preconditioning with cathodal TDCS can flip the "normal" suppressive effect of 1 $\mathrm{Hz}$ rTMS and cause an apparently paradoxical facilitation. Ziemann et al. (1998) demonstrated that the response to rTMS can be influenced by acute deafferentation. A total of $0.1 \mathrm{~Hz}$ rTMS, which was normally ineffective in changing corticospinal excitability, increased the excitability of the projection to the biceps muscle if it was applied during anesthesia of the forearm. Together with the present results, it can be concluded that the plastic changes induced by $1 \mathrm{~Hz}$ rTMS critically depend on the functional state of the stimulated cortex before and at the time of rTMS conditioning.

We propose that differences in the recent history of neuronal activity contribute to the marked interindividual variability of the suppressive effect of $1 \mathrm{~Hz}$ rTMS on corticospinal excitability in healthy subjects (Maeda et al., 2000b). Preconditioning offers a feasible approach to "standardize" the suppressive effect of $1 \mathrm{~Hz}$ rTMS across subjects. In neuropsychiatric diseases, systematic changes in cortical excitability and in the history of neuronal activity before rTMS are likely to alter the conditioning effects of $1 \mathrm{~Hz}$ rTMS. Indeed, rTMS can induce different patterns of plasticity in patients compared with healthy controls (Siebner et al., 1999a,b). Therefore, it is problematic to predict the effects of $1 \mathrm{~Hz}$ rTMS in patients on the basis of studies in healthy subjects.

\section{References}

Abbott LF, Nelson SB (2000) Synaptic plasticity: taming the beast. Nat Neurosci [Suppl] 3:1178-1183.

Abraham WC, Tate WP (1997) Metaplasticity: a new vista across the field of synaptic plasticity. Prog Neurobiol 52:303-323.

Allen CB, Celikel T, Feldman DE (2003) Long-term depression induced by sensory deprivation during cortical map plasticity in vivo. Nat Neurosci 6:291-299.

Asanuma H, Keller A (1991) Neuronal mechanisms of motor learning in mammals. NeuroReport 2:217-224.

Bear MF (1996) A synaptic basis for memory storage in the cerebral cortex. Proc Natl Acad Sci USA 93:13453-13459.

Bear MF, Malenka RC (1994) Synaptic plasticity: LTP and LTD. Curr Opin Neurobiol 4:389-399.

Bienenstock EL, Cooper LN, Munro PW (1982) Theory for the development of neuron selectivity: orientation specificity and binocular interaction in visual cortex. J Neurosci 2:32-48.

Bindman LJ, Lippold OC, Redfearn JWT (1962) Long-lasting changes in the level of electrical activity of the cerebral cortex produced by polarizing currents. Nature 196:584-585.

Bindman LJ, Lippold OC, Redfearn JWT (1964) The action of brief polarising currents on the cerebral cortex of the rat (1) during current flow and (2) in the production of long-lasting after effects. J Physiol (Lond) 172:369-382.
Brighina F, Bisiach E, Oliveri M, Piazza A, La Bua V, Daniele O, Fierro B (2003) $1 \mathrm{~Hz}$ repetitive transcranial magnetic stimulation of the unaffected hemisphere ameliorates contralesional visuospatial neglect in humans. Neurosci Lett 336:131-133.

Chen R, Classen J, Gerloff C, Celnik P, Wassermann EM, Hallett M, Cohen LG (1997) Depression of motor cortex excitability by low-frequency transcranial magnetic stimulation. Neurology 48:1398-1403.

Desai NS, Cudmore RH, Nelson SB, Turrigiano GG (2002) Critical periods for experience-dependent synaptic scaling in visual cortex. Nat Neurosci 5:783-789.

Di Lazzaro V, Oliviero A, Profice P, Insola A, Mazzone P, Tonali P, Rothwell JC (1999) Effects of voluntary contraction on descending volleys evoked by transcranial electrical stimulation over the motor cortex hand area in conscious humans. Exp Brain Res 124:525-528.

Di Lazzaro V, Oliviero A, Mazzone P, Pilato F, Saturno E, Dileone M, Insola A, Tonali PA, Rothwell JC (2002) Short-term reduction of intracortical inhibition in the human motor cortex induced by repetitive transcranial magnetic stimulation. Exp Brain Res 147:108-113.

Fitzgerald PB, Brown TL, Daskalakis ZJ, Chen R, Kulkarni J (2002) Intensity-dependent effects of $1 \mathrm{~Hz}$ rTMS on human corticospinal excitability. Clin Neurophysiol 113:1136-1141.

Gartside IB (1968) Mechanisms of sustained increases in firing rate of neurones in the rat cerebral cortex after polarization: role of protein synthesis. Nature 220:383-384.

Hallett M (2000) Transcranial magnetic stimulation and the human brain. Nature 406:147-150.

Hess G, Donoghue JP (1996) Long-term depression of horizontal connections in rat motor cortex. Eur J Neurosci 8:658-665.

Hilgetag CC, Theoret H, Pascual-Leone A (2001) Enhanced visual spatial attention ipsilateral to rTMS-induced "virtual lesions" of human parietal cortex. Nat Neurosci 4:953-957.

Hoffman RE, Hawkins KA, Gueorguieva R, Boutros NN, Rachid F, Carroll K, Krystal JH (2003) Transcranial magnetic stimulation of left temporoparietal cortex and medication-resistant auditory hallucinations. Arch Gen Psychiatry 60:49-56.

Huang YY, Colino A, Selig DK, Malenka RC (1992) The influence of prior synaptic activity on the induction of long-term potentiation. Science 255:730-733.

Iriki A, Pavlides C, Keller A, Asanuma H (1989) Long-term potentiation in the motor cortex. Science 245:1385-1387.

Iyer MB, Schleper N, Wassermann EM (2003) Priming stimulation enhances the depressant effect of low-frequency repetitive transcranial magnetic stimulation. J Neurosci 23:10867-10872.

Kirkwood A, Rioult MC, Bear MF (1996) Experience-dependent modification of synaptic plasticity in visual cortex. Nature 381:526-528.

Klein E, Kreinin I, Chistyakov A, Koren D, Mecz L, Marmur S, Ben-Shachar D, Feinsod M (1999) Therapeutic efficacy of right prefrontal slow repetitive transcranial magnetic stimulation in major depression: a doubleblind controlled study. Arch Gen Psychiatry 56:315-320.

Knecht S, Ellger T, Breitenstein C, Bernd Ringelstein E, Henningsen $\mathrm{H}$ (2003) Changing cortical excitability with low-frequency transcranial magnetic stimulation can induce sustained disruption of tactile perception. Biol Psychiatry 53:175-179.

Kosslyn SM, Pascual-Leone A, Felician O, Camposano S, Keenan JP, Thompson WL, Ganis G, Sukel KE, Alpert NM (1999) The role of area 17 in visual imagery: convergent evidence from positron emission tomography and rTMS. Science 284:167-170.

Kujirai T, Caramia MD, Rothwell JC, Day BL, Thompson PD, Ferbert A, Wroe S, Asselman P, Marsden CD (1993) Corticocortical inhibition in human motor cortex. J Physiol (Lond) 471:501-519.

Liebetanz D, Nitsche MA, Tergau F, Paulus W (2002) Pharmacological approach to the mechanisms of transcranial DC-stimulation-induced aftereffects of human motor cortex excitability. Brain 125:2238-2247.

Maeda F, Keenan JP, Tormos JM, Topka H, Pascual-Leone A (2000a) Modulation of corticospinal excitability by repetitive transcranial magnetic stimulation. Clin Neurophysiol 111:800-805.

Maeda F, Keenan JP, Tormos JM, Topka H, Pascual-Leone A (2000b) Interindividual variability of the modulatory effects of repetitive transcranial magnetic stimulation on cortical excitability. Exp Brain Res 133:425-430.

Muellbacher W, Ziemann U, Boroojerdi B, Hallett M (2000) Effects of lowfrequency transcranial magnetic stimulation on motor excitability and basic motor behavior. Clin Neurophysiol 111:1002-1007. 
Muellbacher W, Ziemann U, Wissel J, Dang N, Kofler M, Facchini S, Boroojerdi B, Poewe W, Hallett M (2002) Early consolidation in human primary motor cortex. Nature 415:640-644.

Munchau A, Bloem BR, Irlbacher K, Trimble MR, Rothwell JC (2002) Functional connectivity of human premotor and motor cortex explored with repetitive transcranial magnetic stimulation. J Neurosci 22:554-561.

Nitsche MA, Paulus W (2000) Excitability changes induced in the human motor cortex by weak transcranial direct current stimulation. J Physiol (Lond) 527:633-639.

Nitsche MA, Paulus W (2001) Sustained excitability elevations induced by transcranial DC motor cortex stimulation in humans. Neurology 57:1899-1901.

Nitsche MA, Nitsche MS, Klein CC, Tergau F, Rothwell JC, Paulus W (2003) Level of action of cathodal DC polarisation induced inhibition of the human motor cortex. Clin Neurophysiol 114:600-604.

Oldfield RC (1971) The assessment and analysis of handedness: the Edinburgh inventory. Neuropsychologia 9:97-113.

Pascual-Leone A, Valls-Sole J, Wassermann EM, Hallett M (1994) Responses to rapid-rate transcranial magnetic stimulation of the human motor cortex. Brain 117:847-858.

Picconi B, Centonze D, Hakansson K, Bernardi G, Greengard P, Fisone G, Cenci MA, Calabresi P (2003) Loss of bidirectional striatal synaptic plasticity in L-DOPA-induced dyskinesia. Nat Neurosci 6:501-506.

Purpura DP, McMurry JG (1965) Intracellular activities and evoked potential changes during polarization of the motor cortex. J Neurophysiol 28:166-185.

Rioult-Pedotti MS, Friedman D, Donoghue JP (2000) Learning-induced LTP in neocortex. Science 290:533-536.

Romero JR, Anschel D, Sparing R, Gangitano M, Pascual-Leone A (2002) Subthreshold low frequency repetitive transcranial magnetic stimulation selectively decreases facilitation in the motor cortex. Clin Neurophysiol 113:101-107.
Rothwell JC (1997) Techniques and mechanisms of action of transcranial stimulation of the human motor cortex. J Neurosci Methods 74:113-122.

Royer S, Pare D (2003) Conservation of total synaptic weight through balanced synaptic depression and potentiation. Nature 422:518-522.

Sejnowski TJ (1977) Statistical constraints on synaptic plasticity. J Theor Biol 69:385-389.

Siebner HR, Rothwell J (2003) Transcranial magnetic stimulation: new insights into representational cortical plasticity. Exp Brain Res 148:1-16.

Siebner HR, Auer C, Ceballos-Baumann A, Conrad B (1999a) Has repetitive transcranial magnetic stimulation of the primary motor hand area therapeutic application in writer's cramp? Electroencephalogr Clin Neurophysiol [Suppl] 51:265-275.

Siebner HR, Tormos JM, Ceballos-Baumann AO, Auer C, Catala MD, Conrad B, Pascual-Leone A (1999b) Low-frequency repetitive transcranial magnetic stimulation of the motor cortex in writer's cramp. Neurology 52:529-537.

Sommer M, Lang N, Tergau F, Paulus W (2002) Neuronal tissue polarization induced by repetitive transcranial magnetic stimulation? NeuroReport 13:809-811.

Tergau F, Naumann U, Paulus W, Steinhoff BJ (1999) Low-frequency repetitive transcranial magnetic stimulation improves intractable epilepsy. Lancet 353:2209.

Touge T, Gerschlager W, Brown P, Rothwell JC (2001) Are the after-effects of low-frequency rTMS on motor cortex excitability due to changes in the efficacy of cortical synapses? Clin Neurophysiol 112:2138-2145.

Turrigiano GG, Nelson SB (2004) Homeostatic plasticity in the developing nervous system. Nat Rev Neurosci 5:97-107.

Wang H, Wagner JJ (1999) Priming-induced shift in synaptic plasticity in the rat hippocampus. J Neurophysiol 82:2024-2028.

Ziemann U, Corwell B, Cohen LG (1998) Modulation of plasticity in human motor cortex after forearm ischemic nerve block. J Neurosci 18: 1115-1123. 